\title{
Facile One-Pot Synthesis of Quinazoline-2,4-dione Derivatives and Application to Naturally Occurring Alkaloids from Zanthoxylum Arborescens
}

\author{
Xin Li and Yong Rok Lee* \\ School of Chemical Engineering and Technology, Yeungnam University, Gyeongsan 712-749, Korea. *E-mail: yrlee@yu.ac.kr \\ Received April 21, 2011, Accepted May 5, 2011
}

Key Words : Quinazoline-2,4-ones, Anti-hypertensive, Alkaloids

\section{Introduction}

Quinazoline-2,4-diones are one of the important heterocycles $^{1}$ and have been shown to possess pharmacologically interesting properties such as anti-hypertensive, ${ }^{2}$ antidiabetic, ${ }^{3}$ and immunosuppressive activities. ${ }^{4}$ Among these, synthetic pelanserine (TR2515) (1) ${ }^{5}$ is a well established potent anti-hypertensive, having activity comparable to ketanserin, ${ }^{6}$ which is an anti-hypertensive agent used clinically. As alkaloids containing the quinazoline-2,4-dione moiety, compounds $\mathbf{2}$ and $\mathbf{3}$ were isolated from Zanthoxylum arborescens (Figure 1). ${ }^{7}$ This wide range of biological activities has stimulated interest in new approaches for the synthesis of quinazoline-2,4-dione derivatives.

Several synthetic approaches to quinazoline-2,4-dione derivatives have been reported. ${ }^{8}$ The general methods include a four-step reaction starting from phthalate monomethyl ester $^{9}$ and one-step palladium-catalyzed urea arylationintramolecular ester amidation. ${ }^{10}$ There is still a demand for a more convenient and efficient synthetic method that can efficiently provide quinazoline-2,4-dione derivatives. We report herein a convenient one-pot synthesis of quinazoline2,4-dione derivatives. We also report on the synthesis of the naturally occurring alkaloids $\mathbf{2}$ and $\mathbf{3}$.

\section{Results and Discussion}

A two-step reaction for the synthesis of quinazoline-2,4dione derivatives starting from isatoic anhydride and amines has already been described. ${ }^{11}$ However, a one-pot approach for the synthesis of quinazoline-2,4-dione derivatives from commercially available isatoic anhydride has not been reported. Direct reaction of isatoic anhydride (4) with tryptamine (5) in THF at room temperature for $5 \mathrm{~h}$ afforded aminobenzamide 6 in $80 \%$ yield (Scheme 1) without any formation of quinazoline-2,4-dione 7. The nucleophilic

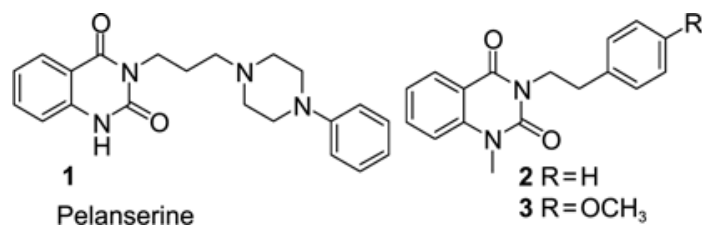

Figure 1. Synthesized and naturally occurring representative quinazoline-2,4-diones. attack of primary amine of $\mathbf{5}$ to carbonyl group on the oxazine ring of isatoic anhydride (4) followed by loss of carbon dioxide produced compound 6. The structural assignment of 6 was confirmed by ${ }^{13} \mathrm{C} \mathrm{NMR}$, which clearly showed a carbonyl peak of amide group at $168.9 \mathrm{ppm}$. To give the quinazoline-2,4-dione moiety, reaction of isatoic anhydride (4) and tryptamine (5) with triphosgene was next attempted. Treatment of isatoic anhydride (4) with both tryptamine (5) and triphosgene in the presence of $\mathrm{K}_{2} \mathrm{CO}_{3}$ in $\mathrm{THF}$ at room temperature for $20 \mathrm{~h}$ gave the product 7 in only $20 \%$ yield. However, treatment of $\mathbf{4}$ with $\mathbf{5}$ in THF at room temperature for $5 \mathrm{~h}$ followed by further reaction by addition of triphosgene and $\mathrm{K}_{2} \mathrm{CO}_{3}$ at room temperature for $15 \mathrm{~h}$ provided 7 in increased yield (73\%). The structure of 7 was easily confirmed by ${ }^{1} \mathrm{H}$ NMR analysis and direct comparison with reported data. ${ }^{12}$

In order to extend the utility of this methodology, further reactions of isatoic anhydride (4) and several primary amines with triphosgene in the presence of $\mathrm{K}_{2} \mathrm{CO}_{3}$ using conditions described above were next attempted to synthesize a variety of quinazoline-2,4-dione derivatives. The results are summarized in Table 1. First, the reaction of isatoic anhydride and aliphatic amines with a long chain or cyclic ring was examined. The reaction of $\mathbf{4}$ with butylamine afforded $\mathbf{8}$ in $50 \%$ yield, whereas that with 1-hexylamine gave 9 in $71 \%$ yield (entries 1 and 2). Similarly, the reaction of 4-phenylbutylamine, 2-piperidinoethylamine, 2-morpholinoethylamine, and cyclohexylmethylamine having a ring provided the desired products 10-13 in 55, 44, 52, and 53\% yield (entries

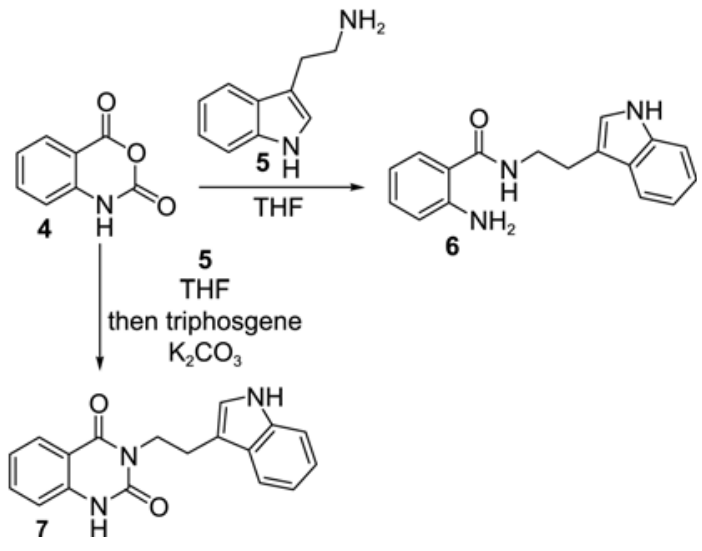

Scheme 1 
Table 1. Synthesis of a variety of quinazoline-2,4-diones starting from isatoic anhydride (4)

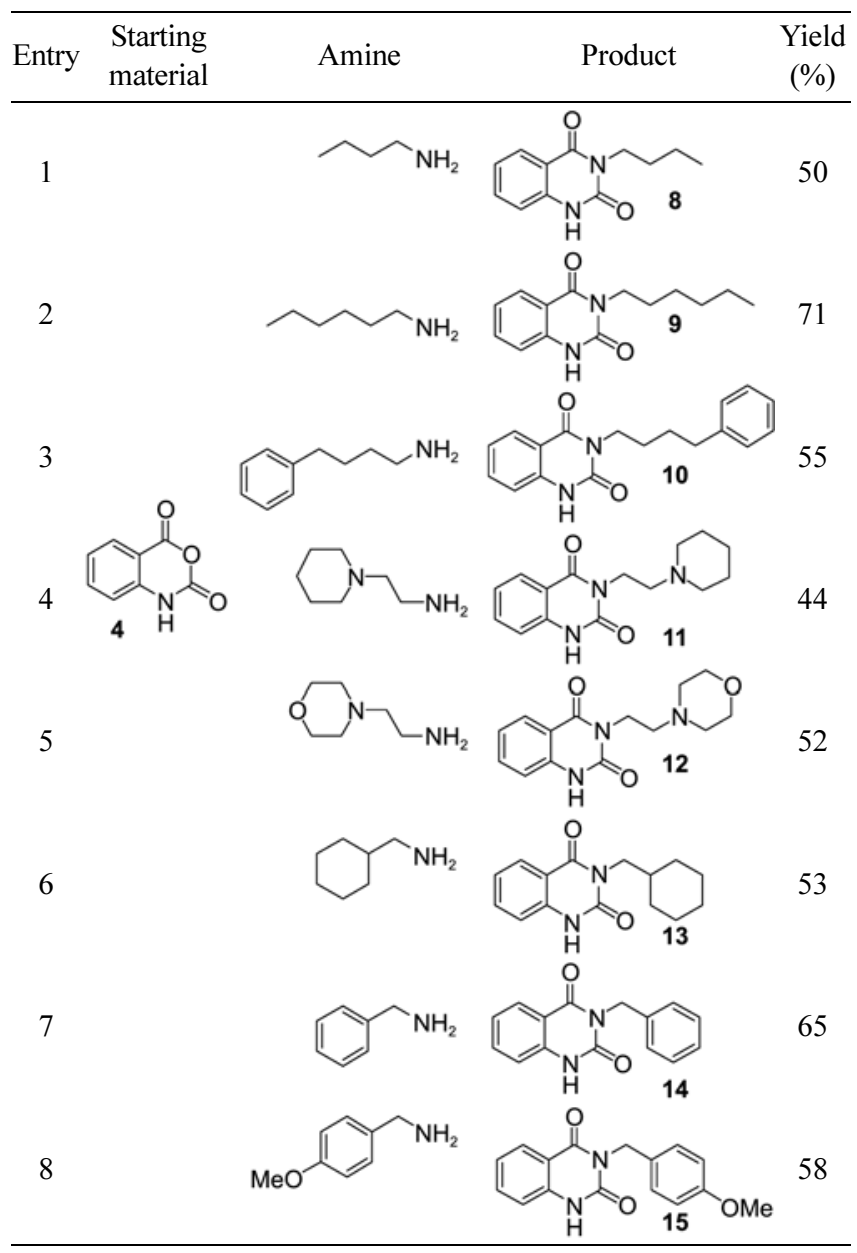

3-6), respectively. Reactions with benzylamine and 4-methoxybenzylamine gave compounds 14 and 15 in 65 and 58\% yield, respectively (entries 7 and 8). These reactions provided a rapid synthetic route to quinazoline-2,4-dione derivatives with a variety of substituents at the 3-position of the quinazolinedione moiety.

The formation of 7 may be explained as shown in Scheme 2. Amine 5 was first reacted with isatoic anhydride (4) to give the aminobenzamide 6 . The ring opening of $\mathbf{4}$ with amines has already been described by another group. ${ }^{13}$ The aminobenzamide 6 was cyclized using triphosgene in the presence of a base through the intermediate $\mathbf{1 6}$ to give product 7. The existence of the isocyanate intermediate formed from the reaction of aminobenzamide and triphosgene has also been proven by Eckert. ${ }^{14}$

As an application of this methodology, the concise synthesis of naturally occurring alkaloids $\mathbf{2}$ and $\mathbf{3}$ was attempted (Scheme 3). Reaction of $\mathbf{4}$ with phenethylamine in THF at room temperature for $5 \mathrm{~h}$ followed by further reaction with triphosgene in the presence of 5 equivalents of $\mathrm{K}_{2} \mathrm{CO}_{3}$ at room temperature for $15 \mathrm{~h}$ afforded $\mathbf{1 7}$ in $78 \%$ yield, whereas that with 4-methoxyphenethylamine gave $\mathbf{1 8}$ in $81 \%$ yield. Treatment of $\mathbf{1 7}$ and $\mathbf{1 8}$ with methyl iodide in the presence of $\mathrm{K}_{2} \mathrm{CO}_{3}$ in refluxing acetone for $5 \mathrm{~h}$ gave the
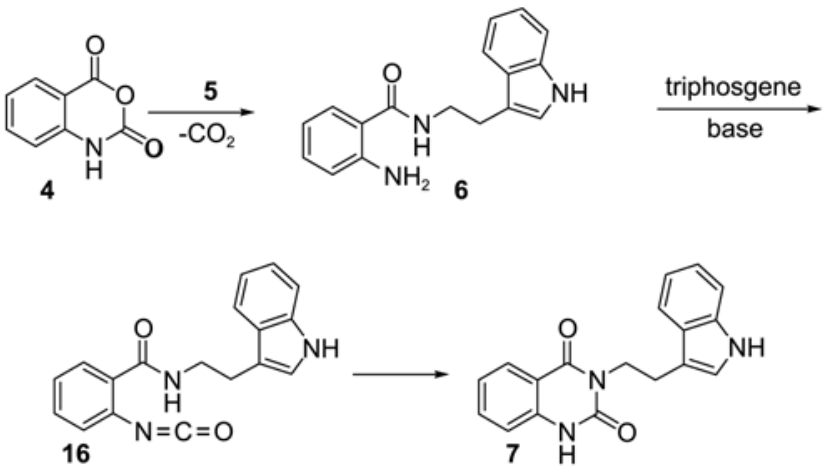

Scheme 2<smiles>O=c1[nH]c2ccccc2c(=O)o1</smiles>

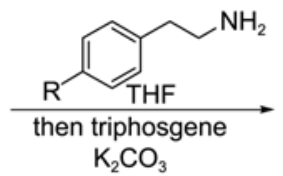<smiles>[R]c1ccc(CCn2c(=O)[nH]c3ccccc3c2=O)cc1</smiles>

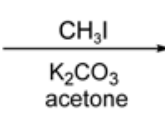<smiles>[R]c1ccc(CCn2c(=O)c3ccccc3n(C[R]([H])=O)c2=O)cc1</smiles>

Scheme 3

expected products $\mathbf{2}$ and $\mathbf{3}$ in 95 and $83 \%$ yield, respectively. The spectroscopic data for synthetic materials $\mathbf{2}$ and $\mathbf{3}$ are in good agreement with the data reported for the natural products. $^{7}$

In conclusion, we have described a one-pot synthesis of biologically interesting quinazoline-2,4-dione derivatives starting from isatoic anhydride with primary amines and subsequent addition of triphosgene under a base. As an application of this methodology, naturally occurring alkaloids $\mathbf{2}$ and $\mathbf{3}$ were synthesized starting from isatoic anhydride in 2-step.

\section{Experimental Section}

All the experiments were carried out in a nitrogen atmosphere. Merck precoated silica gel plates (Art. 5554) with a fluorescent indicator were used for analytical TLC. Flash column chromatography was performed using silica gel 9385 (Merck). The ${ }^{1} \mathrm{H}$ NMR and ${ }^{13} \mathrm{C}$ NMR spectra were recorded on a Bruker Model ARX (300 and $75 \mathrm{MHz}$, respectively) spectrometer in $\mathrm{CDCl}_{3}$ or DMSO- $d_{6}$. The IR spectra were recorded on a Jasco FTIR 5300 spectrophotometer.

2-Amino- $\boldsymbol{N}$-[2-(1 H-indol-3-yl)ethyl]benzamide (6). To a solution of isatoic anhydride (163 mg, $1.0 \mathrm{mmol})$ in THF (15 $\mathrm{mL})$ was added tryptamine $(1.1 \mathrm{mmol})$. The mixture was stirred at room temperature for $5 \mathrm{~h}$. The reaction mixture 
was quenched by the addition of saturated $\mathrm{NH}_{4} \mathrm{Cl}$ solution $(50 \mathrm{~mL})$ and extracted with ethyl acetate $(50 \mathrm{~mL} \times 3)$. The organic layer was washed with water $(40 \mathrm{~mL})$, dried $\left(\mathrm{MgSO}_{4}\right)$, and evaporated under reduced pressure to give yellow solids. The solids were recrystallized by ethanol to give 6 (223 mg, 80\%) as a solid; $\mathrm{mp} 159-160{ }^{\circ} \mathrm{C}$; ${ }^{1} \mathrm{H}$ NMR $\left(300 \mathrm{MHz}, \mathrm{DMSO}-d_{6}\right) \delta 10.82(1 \mathrm{H}, \mathrm{s}), 8.35(1 \mathrm{H}, \mathrm{t}, J=5.5$ $\mathrm{Hz}), 7.59$ (1H, d, $J=7.8 \mathrm{~Hz}), 7.46(1 \mathrm{H}, \mathrm{d}, J=7.8 \mathrm{~Hz}), 7.35$ $(1 \mathrm{H}, \mathrm{d}, J=8.1 \mathrm{~Hz}), 7.19-6.97(4 \mathrm{H}, \mathrm{m}), 6.70(1 \mathrm{H}, \mathrm{d}, J=7.5$ $\mathrm{Hz}), 6.50(1 \mathrm{H}, \mathrm{dd}, J=7.5,7.0 \mathrm{~Hz}), 6.42(2 \mathrm{H}, \mathrm{s}), 3.54(2 \mathrm{H}, \mathrm{t}$, $J=7.5 \mathrm{~Hz}), 2.94(2 \mathrm{H}, \mathrm{t}, J=7.5 \mathrm{~Hz}) ;{ }^{13} \mathrm{C} \mathrm{NMR}(300 \mathrm{MHz}$, DMSO- $\left.d_{6}\right) \delta 168.9,149.7,136.3,131.6,128.1,127.4,122.7$, 121.0, 118.4, 118.3, 116.4, 115.0, 114.7, 112.1, 111.5, 39.9, 25.3; IR (KBr) 3409, 3056, 2927, 1630, 1581, 1518, 1426, $1276,1245,1171,1096,746 \mathrm{~cm}^{-1}$.

Typical Procedures for the Synthesis of Quinazoline2,4-diones 7-15 and 17-18. To a solution of isatoic anhydride $(163 \mathrm{mg}, 1.0 \mathrm{mmol})$ in THF $(15 \mathrm{~mL})$ was added amines $(1.1 \mathrm{mmol})$. The mixture was stirred at room temperature for $5 \mathrm{~h}$. Then triphosgene $(296 \mathrm{mg}, 1.0 \mathrm{mmol})$ and $\mathrm{K}_{2} \mathrm{CO}_{3}(690 \mathrm{mg}, 5.0 \mathrm{mmol})$ was added and the resulting mixture was further stirred for $15 \mathrm{~h}$ to complete the reactions. The reaction mixture was quenched by the addition of saturated $\mathrm{NH}_{4} \mathrm{Cl}$ solution $(50 \mathrm{~mL})$ and extracted with ethyl acetate $(50 \mathrm{~mL} \times 3)$. The organic layer was washed with water $(40 \mathrm{~mL})$, dried $\left(\mathrm{MgSO}_{4}\right)$, and evaporated under reduced pressure to give yellow solids. The solids were recrystallized by ethanol to give pure products 7-15 and 17-18.

3-[2-(1H-Indol-3-yl)ethyl]-1 $H, 3 H$-quinazoline-2,4-dione (7): Yield 73\%; mp 306-307 ${ }^{\circ} \mathrm{C}$; ${ }^{1} \mathrm{H}$ NMR (300 MHz, DMSO$\left.d_{6}\right) \delta 11.3(1 \mathrm{H}, \mathrm{s}), 10.80(1 \mathrm{H}, \mathrm{s}), 7.98(1 \mathrm{H}, \mathrm{d}, J=7.5 \mathrm{~Hz})$, $7.69(1 \mathrm{H}, \mathrm{d}, J=7.5 \mathrm{~Hz}), 7.66(1 \mathrm{H}, \mathrm{dd}, J=7.8,7.0 \mathrm{~Hz}), 7.35$ $(1 \mathrm{H}, \mathrm{d}, J=7.8 \mathrm{~Hz}), 7.24-7.19(3 \mathrm{H}, \mathrm{m}), 7.08(1 \mathrm{H}, \mathrm{dd}, J=7.5$, $7.0 \mathrm{~Hz}), 7.00(1 \mathrm{H}, \mathrm{dd}, J=7.5,7.0 \mathrm{~Hz}), 4.17(2 \mathrm{H}, \mathrm{t}, J=8.1$ $\mathrm{Hz}), 2.99(2 \mathrm{H}, \mathrm{t}, J=8.1 \mathrm{~Hz}) ;{ }^{13} \mathrm{C}$ NMR (300 MHz, DMSO$\left.d_{6}\right) \delta 161.8,150.0,139.2,136.2,134.6,127.0,122.5,122.1$, 120.7, 118.1, 114.9, 113.7, 111.1, 111.0, 40.5, 23.3; IR (KBr) 3366,3063 , 1706, 1645, 1448, 1349, 1281, 1104, 1003, 740 $\mathrm{cm}^{-1}$.

3-Butyl-1H,3H-quinazoline-2,4-dione (8): Yield 50\%; mp 156-156 ${ }^{\circ} \mathrm{C} ;{ }^{1} \mathrm{H}$ NMR $\left(300 \mathrm{MHz}, \mathrm{CDCl}_{3}\right) \delta 10.82(1 \mathrm{H}$, s), $8.10(1 \mathrm{H}, \mathrm{d}, J=7.8 \mathrm{~Hz}), 7.57(1 \mathrm{H}, \mathrm{t}, J=7.8 \mathrm{~Hz}), 7.21-$ $7.12(2 \mathrm{H}, \mathrm{m}), 4.08(2 \mathrm{H}, \mathrm{t}, J=7.5 \mathrm{~Hz}), 1.74-1.59(2 \mathrm{H}, \mathrm{m})$, 1.47-1.35 (2H, m), 0.95(3H, t, $J=7.2 \mathrm{~Hz}) ;{ }^{13} \mathrm{C}$ NMR $(75$ $\left.\mathrm{MHz}, \mathrm{CDCl}_{3}\right) \delta 162.4,152.4,138.7,134.8,128.2,123.2$, $115.1,114.6,40.8,30.0,20.2$, 13.8; IR (KBr) 3194, 3065, $2955,1715,1639,1493,1450,1410,1345,1274,1078,950$, $760 \mathrm{~cm}^{-1}$.

3-Hexyl-1H,3H-quinazoline-2,4-dione (9): Yield 71\%; mp 152-154 ${ }^{\circ} \mathrm{C} ;{ }^{1} \mathrm{H}$ NMR $\left(300 \mathrm{MHz}, \mathrm{CDCl}_{3}\right) \delta 9.97(1 \mathrm{H}, \mathrm{s})$, $8.12(1 \mathrm{H}, \mathrm{d}, J=7.8 \mathrm{~Hz}), 7.59(1 \mathrm{H}, \mathrm{dd}, J=8.1,7.8 \mathrm{~Hz}), 7.21$ $(1 \mathrm{H}, \mathrm{dd}, J=8.1,7.8 \mathrm{~Hz}), 7.08(1 \mathrm{H}, \mathrm{d}, J=8.1 \mathrm{~Hz}), 4.06(2 \mathrm{H}$, $\mathrm{t}, J=7.5 \mathrm{~Hz}), 1.75-1.65(2 \mathrm{H}, \mathrm{m}), 1.42-1.30(6 \mathrm{H}, \mathrm{m}), 0.87$ $(3 \mathrm{H}, \mathrm{t}, J=7.2 \mathrm{~Hz}) ;{ }^{13} \mathrm{C} \mathrm{NMR}\left(75 \mathrm{MHz}, \mathrm{CDCl}_{3}\right) \delta 162.3$, $151.9,138.5,134.9,128.4,123.3,114.9,114.4,41.1,31.5$, 27.8, 26.6, 22.5, 14.1; IR (KBr) 3440, 3062, 2933, 2359, $1714,1660,1454,1282,1081,837,756 \mathrm{~cm}^{-1}$.
3-(4-Phenylbutyl)-1H,3H-quinazoline-2,4-dione (10): Yield 55\%; mp 97-98 ${ }^{\circ} \mathrm{C}$; ${ }^{1} \mathrm{H}$ NMR $\left(300 \mathrm{MHz}, \mathrm{CDCl}_{3}\right) \delta$ $10.61(1 \mathrm{H}, \mathrm{s}), 8.13(1 \mathrm{H}, \mathrm{d}, J=7.8 \mathrm{~Hz}), 7.58(1 \mathrm{H}, \mathrm{dd}, J=8.1$, $7.2 \mathrm{~Hz}), 7.27-7.10(7 \mathrm{H}, \mathrm{m}), 4.14(2 \mathrm{H}, \mathrm{t}, J=6.9 \mathrm{~Hz}), 2.69$ $(2 \mathrm{H}, \mathrm{t}, J=6.9 \mathrm{~Hz}), 1.79-1.73(4 \mathrm{H}, \mathrm{m}) ;{ }^{13} \mathrm{C} \mathrm{NMR}(75 \mathrm{MHz}$, $\left.\mathrm{CDCl}_{3}\right) \delta 162.3,152.4,142.2,138.7,134.9,128.4,128.3$, 128.2, 125.7, 123.3, 115.0, 114.7, 40.8, 35.6, 28.7, 27.6; IR (KBr) 3195, 2941, 1720, 1635, 1448, 1358, 1269, 1111, $1056,755 \mathrm{~cm}^{-1}$.

3-[2-(Piperidin-1-yl) ethyl]-1H,3H-quinazoline-2,4dione (11): Yield 44\%; mp 187-189 ${ }^{\circ} \mathrm{C} ;{ }^{1} \mathrm{H}$ NMR (300 MHz, $\left.\mathrm{CDCl}_{3}\right) \delta 10.46(1 \mathrm{H}, \mathrm{s}), 8.02(1 \mathrm{H}, \mathrm{d}, J=8.1 \mathrm{~Hz}), 7.53(1 \mathrm{H}$, $\mathrm{dd}, J=8.1,7.5 \mathrm{~Hz}), 7.14(1 \mathrm{H}, \mathrm{dd}, J=8.1,7.5 \mathrm{~Hz}), 7.04(1 \mathrm{H}$, $\mathrm{d}, J=8.1 \mathrm{~Hz}), 4.24(2 \mathrm{H}, \mathrm{t}, J=7.2 \mathrm{~Hz}), 2.69(2 \mathrm{H}, \mathrm{t}, J=7.2$ $\mathrm{Hz}), 2.64-2.52$ (4H, m), 1.66-1.54 (4H, m), 1.47-1.38 (2H, $\mathrm{m}) ;{ }^{13} \mathrm{C} \mathrm{NMR}\left(75 \mathrm{MHz}, \mathrm{CDCl}_{3}\right) \delta 162.3,151.8,138.7$, $134.8,128.3,123.2,114.8,114.5,56.2,54.7,38.0,25.8$, 24.3; IR (KBr) 3442, 3062, 2931, 1720, 1658, 1449, 1294, $1112,1044,832,755 \mathrm{~cm}^{-1}$.

3-[2-(Morpholin-4-yl)ethyl]-1H,3H-quinazoline-2,4-dione (12): Yield 52\%; mp $198-203{ }^{\circ} \mathrm{C}$; ${ }^{1} \mathrm{H}$ NMR $(300 \mathrm{MHz}$, $\left.\mathrm{CDCl}_{3}\right) \delta 10.04(1 \mathrm{H}, \mathrm{s}), 8.08(1 \mathrm{H}, \mathrm{d}, J=7.8 \mathrm{~Hz}), 7.57(1 \mathrm{H}$, $\mathrm{dd}, J=7.8,7.2 \mathrm{~Hz}), 7.20(1 \mathrm{H}, \mathrm{dd}, J=8.0,7.2 \mathrm{~Hz}), 7.04(1 \mathrm{H}$, d, $J=8.1 \mathrm{~Hz}), 4.23(2 \mathrm{H}, \mathrm{t}, J=6.6 \mathrm{~Hz}), 3.68(4 \mathrm{H}, \mathrm{t}, J=4.5$ $\mathrm{Hz}), 2.70$ (2H, t, $J=6.6 \mathrm{~Hz}), 2.64-2.54(4 \mathrm{H}, \mathrm{m}) ;{ }^{13} \mathrm{C} \mathrm{NMR}$ $\left(75 \mathrm{MHz}, \mathrm{CDCl}_{3}\right) \delta 162.3,151.8,138.5,135.0,128.4,123.4$, 114.8, 114.6, 67.0, 56.0, 53.8, 37.7; IR (KBr) 3439, 3065, $2931,1718,1647,1451,1283,1116,862,759 \mathrm{~cm}^{-1}$.

3-(Cyclohexylmethyl)-1H,3H-quinazoline-2,4-dione (13): Yield 53\%; mp 209-212 ${ }^{\circ} \mathrm{C} ;{ }^{1} \mathrm{H}-\mathrm{NMR}\left(300 \mathrm{MHz}, \mathrm{CDCl}_{3}\right) \delta$ $10.40(1 \mathrm{H}, \mathrm{s}), 8.12(1 \mathrm{H}, \mathrm{d}, J=7.8 \mathrm{~Hz}), 7.59(1 \mathrm{H}, \mathrm{dd}, J=7.8$, $7.2 \mathrm{~Hz}), 7.02(1 \mathrm{H}, \mathrm{dd}, J=8.1,7.2 \mathrm{~Hz}), 7.11(1 \mathrm{H}, \mathrm{d}, J=8.1$ $\mathrm{Hz}), 3.96$ (2H, d, $J=7.2 \mathrm{~Hz}), 1.95-1.82(1 \mathrm{H}, \mathrm{m}), 1.76-1.63$ $(5 \mathrm{H}, \mathrm{m}), 1.28-1.10(5 \mathrm{H}, \mathrm{m}) ;{ }^{13} \mathrm{C} \mathrm{NMR}\left(75 \mathrm{MHz}, \mathrm{CDCl}_{3}\right) \delta$ 162.7, 162.3, 152.5, 138.7, 134.9, 128.5, 123.3, 115.0, 114.7, 46.8, 36.6, 30.9, 26.4, 25.9; IR (KBr) 3440, 3195, 2919, $1719,1638,1445,1269,1111,952,758 \mathrm{~cm}^{-1}$.

3-Benzyl-1H,3H-quinazoline-2,4-dione (14): Yield 65\%; mp 214-236 ${ }^{\circ} \mathrm{C}$; ${ }^{1} \mathrm{H}$ NMR $\left(300 \mathrm{MHz}, \mathrm{CDCl}_{3}\right) \delta 10.07(1 \mathrm{H}$, s), $8.13(1 \mathrm{H}, \mathrm{d}, J=7.8 \mathrm{~Hz}), 7.59-7.49(3 \mathrm{H}, \mathrm{m}), 7.30-7.17$ $(4 \mathrm{H}, \mathrm{m}), 7.03(1 \mathrm{H}, \mathrm{d}, J=8.1 \mathrm{~Hz}), 5.27(2 \mathrm{H}, \mathrm{s}) ;{ }^{13} \mathrm{C} \mathrm{NMR}(75$ $\left.\mathrm{MHz}, \mathrm{CDCl}_{3}\right) \delta 162.4,151.0,139.2,137.0,134.5,128.4$, $128.1,127.9,127.1,122.5,115.1,114.2,43.7$; IR (KBr) 3205, 3074, 2943, 1313, 1662, 1491, 1449, 1406, 1350, 963, $760 \mathrm{~cm}^{-1}$.

3-(4-Methoxybenzyl)-1 $\boldsymbol{H , 3 H}$-quinazoline-2,4-dione (15): Yield 58\%; mp 210-216 ${ }^{\circ} \mathrm{C} ;{ }^{1} \mathrm{H}$ NMR (300 MHz, $\left.\mathrm{CDCl}_{3}\right) \delta$ $10.29(1 \mathrm{H}, \mathrm{s}), 8.12(1 \mathrm{H}, \mathrm{d}, J=7.5 \mathrm{~Hz}), 7.56(1 \mathrm{H}, \mathrm{t}, J=7.5$, $7.0 \mathrm{~Hz}), 7.48(2 \mathrm{H}, \mathrm{d}, J=7.8 \mathrm{~Hz}), 7.19(1 \mathrm{H}, \mathrm{dd}, J=7.8,7.0$ $\mathrm{Hz}), 7.05(1 \mathrm{H}, \mathrm{d}, J=7.8 \mathrm{~Hz}), 6.86(2 \mathrm{H}, \mathrm{d}, J=7.8 \mathrm{~Hz}), 5.21$ $(2 \mathrm{H}, \mathrm{s}), 3.74(3 \mathrm{H}, \mathrm{s}) ;{ }^{13} \mathrm{C} \mathrm{NMR}\left(75 \mathrm{MHz}, \mathrm{CDCl}_{3}\right) \delta 162.4$, $159.2,152.3,138.6,135.0,130.5,129.2,128.5,123.4$, 115.0, 114.8, 113.8, 55.2, 43.7; IR (KBr) 3440,3070, 2929, $1713,1654,1509,1448,1251,1170,1107,1036,823,760$ $\mathrm{cm}^{-1}$.

3-Phenethyl-1H,3H-quinazoline-2,4-dione (17): Yield 78\%; mp 174-175 ${ }^{\circ} \mathrm{C}$; ${ }^{1} \mathrm{H}$ NMR (300 MHz, $\left.\mathrm{CDCl}_{3}\right) \delta 10.45$ 
$(1 \mathrm{H}, \mathrm{s}), 8.14(1 \mathrm{H}, \mathrm{d}, J=7.8 \mathrm{~Hz}), 7.61(1 \mathrm{H}, \mathrm{dd}, J=8.1,7.8$ $\mathrm{Hz}), 7.35-7.17(6 \mathrm{H}, \mathrm{m}), 7.12(1 \mathrm{H}, \mathrm{d}, J=8.1 \mathrm{~Hz}), 4.35-4.30$ $(2 \mathrm{H}, \mathrm{m}), 3.10-3.00(2 \mathrm{H}, \mathrm{m}) ;{ }^{13} \mathrm{C}$ NMR $\left(75 \mathrm{MHz}, \mathrm{CDCl}_{3}\right) \delta$ 162.2 , 152.0, 138.7, 138.6, 135.0, 128.9, 128.5, 128.4, 126.5, 123.4, 115.1, 114.7, 42.3, 34.1; IR (KBr) 3063, 2937, 1716, $1658,1493,1451,1411,1352,1282,1168,1114,1004,824$, $760 \mathrm{~cm}^{-1}$.

3-(4-Methoxyphenethyl)-1 $H, 3 H$-quinazoline-2,4-dione (18): Yield 81\%; mp 224-228 ${ }^{\circ} \mathrm{C}$; ${ }^{1} \mathrm{H}$ NMR (300 MHz, $\left.\mathrm{CDCl}_{3}\right) \delta 10.73(1 \mathrm{H}, \mathrm{s}), 7.87(1 \mathrm{H}, \mathrm{d}, J=8.1 \mathrm{~Hz}), 7.34(1 \mathrm{H}, \mathrm{t}$, $J=8.4 \mathrm{~Hz}), 7.04-6.94$ (4H, m), $6.63(2 \mathrm{H}, \mathrm{d}, J=8.7 \mathrm{~Hz})$, 4.05-3.99 (2H, m), $3.58(3 \mathrm{H}, \mathrm{s}), 2.75-2.70(2 \mathrm{H}, \mathrm{m}) ;{ }^{13} \mathrm{C}$ NMR (75 MHz, $\left.\mathrm{CDCl}_{3}\right) \delta 162.1,157.7,150.4,139.1,134.2$, $130.4,129.4,127.4,122.1,114.8,113.9,113.4,54.7,41.7$, 32.6; IR (KBr) 3459, 2927, 1723, 1655, 1512, 1452, 1410, $1289,1246,1177,1122,1032,826,754 \mathrm{~cm}^{-1}$.

General Procedure for Synthesis of 2 and 3. Methyl iodide $(85 \mathrm{mg}, 0.6 \mathrm{mmol})$ in acetone $(1.0 \mathrm{~mL})$ was added to a solution of compound $17(133 \mathrm{mg}, 0.5 \mathrm{mmol})$ or $\mathbf{1 8}(148$ $\mathrm{mg}, 0.5 \mathrm{mmol})$ and potassium carbonate $(345 \mathrm{mg}, 2.5 \mathrm{mmol})$ in acetone $(10 \mathrm{~mL})$. The reaction mixture was stirred in refluxing acetone for $5 \mathrm{~h}$. The solvent was evaporated under reduced pressure. The residue was treated with water, acidified with a saturated aqueous $\mathrm{NH}_{4} \mathrm{Cl}$ solution $(50 \mathrm{~mL})$, and extracted with ethyl acetate $(50 \mathrm{~mL} \times 3)$. The combined organic layer were washed with brine $(50 \mathrm{~mL})$, dried over $\mathrm{MgSO}_{4}$, filtered, and evaporated under reduced pressure. The resulting residue was purified by flash column chromatography on silica gel to give $\mathbf{2}$ or $\mathbf{3}$.

1-Methyl-3-phenethy-1H,3H-quinazoline-2,4-dione (2): Yield 95\%; mp 101-102 ${ }^{\circ} \mathrm{C} ;{ }^{1} \mathrm{H}$ NMR (300 MHz, $\left.\mathrm{CDCl}_{3}\right) \delta$ $8.21(1 \mathrm{H}, \mathrm{dd}, J=7.8,1.5 \mathrm{~Hz}), 7.67-7.62(1 \mathrm{H}, \mathrm{m}), 7.34-7.15$ $(7 \mathrm{H}, \mathrm{m}), 4.32-4.26(2 \mathrm{H}, \mathrm{m}), 3.58(3 \mathrm{H}, \mathrm{m}), 3.00-2.94(2 \mathrm{H}$, $\mathrm{m}) ;{ }^{13} \mathrm{C} \mathrm{NMR}\left(75 \mathrm{MHz}, \mathrm{CDCl}_{3}\right) \delta 161.5,150.8,140.5$, 138.6, 134.9, 128.9, 128.8, 128.4, 126.4, 122.9, 115.6, 113.4, 43.2, 34.0, 30.6; IR (KBr) 3029, 2942, 1700, 1654, 1608, 1484, 1427, 1397, 1351, 1317, 1257, 1142, 1031, $759 \mathrm{~cm}^{-1}$.

1-Methyl-3-(4-Methoxyphenethyl)- $\mathbf{H}, 3 \mathrm{H}$-quinazoline2,4-dione (3): Yield 83\%; mp 135-136 ${ }^{\circ} \mathrm{C}$; ${ }^{1} \mathrm{H}$ NMR (300 $\left.\mathrm{MHz}, \mathrm{CDCl}_{3}\right) \delta 8.20(1 \mathrm{H}, \mathrm{dd}, J=7.8,1.2 \mathrm{~Hz}), 7.64(1 \mathrm{H}$, $\mathrm{ddd}, J=8.4,7.2,1.2 \mathrm{~Hz}), 7.26-7.20(3 \mathrm{H}, \mathrm{m}), 7.17(1 \mathrm{H}, \mathrm{d}, J=$ $8.4 \mathrm{~Hz}), 6.82(2 \mathrm{H}, \mathrm{d}, \mathrm{J}=8.7 \mathrm{~Hz}), 4.27-4.22(2 \mathrm{H}, \mathrm{m}), 3.79$
$(3 \mathrm{H}, \mathrm{s}), 3.57$ (3H, s), 2.93-2.89 (2H, m); ${ }^{13} \mathrm{C}$ NMR (75 MHz, $\left.\mathrm{CDCl}_{3}\right) \delta 161.5,158.3,150.9,140.5,135.0,130.7,129.9$, $128.9,122.9,115.6,113.9,113.4,55.2,43.4,33.1,30.6$; IR (KBr) 3442, 3032, 2966, 2930, 1696, 1653, 1612, 1508, $1483,1428,1350,1243,1175,1127,1031,834,755 \mathrm{~cm}^{-1}$.

Acknowledgments. This work was supported by grant No. RTI04-01-04 from the Regional Technology Innovation Program of the Ministry of Knowledge Economy (MKE).

\section{References}

1. Undheim, K.; Benneche, T. In Comprehensive Heterocyclic Chemistry II; Katritzky, A. R., Rees, C. W., Scriven, E. F. V., Eds.; Elsevier: Oxford, UK, 1996; Vol. 6, p 93.

2. Ismail, M. A. H.; Barker, S.; El Ella, D. A. A.; Abouzid, K. A. M.; Toubar, R. A.; Todd, M. H. J. Med. Chem. 2006, 49, 1526.

3. Goto, S.; Tsuboi, H.; Kagara, K. Chem. Exp. 1993, 8, 761.

4. Buckley, G. M.; Davies, N.; Dyke, H. J.; Gilbert, P. J.; Hannah, D. R.; Haughan, A. F.; Hunt, C. A.; Pitt, W. R.; Profit, R. H.; Ray, N. C.; Richard, M. D.; Sharpe, A.; Taylor, A. J.; Whitworth, J. M.; Williams, S. C. Bioorg. Med. Chem. Lett. 2005, 15, 751.

5. Hayao, S.; Havera, M. J.; Strycker, W. G.; Leipzig, T. J.; Kulp, R. A.; Hartzler, H. E. J. Med. Chem. 1965, 8, 807.

6. (a) Leysen, J. E.; Niemegeers, J. E.; Neuten, J. M.; Lauuron, P. M. Mol. Pharmacol. 1982, 21, 301. (b) Darchen, F.; Scherman, D.; Laduron, P. M.; Henry, J. P. Mol. Pharmacol. 1988, 33, 672.

7. Dreyer, D. L.; Bayer, R. C. Phytochemisry 1980, 19, 935.

8. Jin, H. Z.; Du, J. L.; Zhang, W. D.; Chen, H. S.; Lee, J. H.; Lee, J. J. J. Asian Nat. Prod. Res. 2007, 9, 685.

9. (a) Nikpour, F.; paibast, T. Chem. Lett. 2005, 34. (b) Tran, T. P.; Ellsworth, E. L.; Watson, B. M.; Sanchez, J. P.; Showalter, H. D. H.; Rubin, J. R.; Stier, M. A.; Yip, J.; Nguyen, D. Q.; Bird, P.; Singh, R. J. Heterocycl. Chem. 2005, 42, 669. (c) Makino, S.; Suzuki, N.; Nakanishi, E.; Tsuji, T. Synlett 2001, 333. (d) Goto, S.; Tsuboi, H.; Kanoda, M.; Mukai, K.; Kagara, K. Org. Proc. Res. Dev. 2003, 7, 700. (e) Shao, H.; Colucci, M.; Tong, S.; Zhang, H.; Castelhano, A. L. Tetrahedron Lett. 1998, 39, 7235.

10. (a) Bonjouklian, R.; Ruden, R. A. J. Org. Chem. 1977, 42, 4095. (b) Chantegrel, B.; Gelin, R. A. Synthesis 1981, 315.

11. Willis, M. C.; Snell, R. H.; Fletcher, A. J.; Woodward, R. L. Org. Lett. 2006, 8, 5089

12. Bergman, J.; Bergman, S. J. Org. Chem. 1985, 50, 1246.

13. Kaneko, C.; Chiba, T.; Kasai, K.; Miwa, C. Heterocycles 1985, 23, 1385.

14. Chen, Z.; Hu, G.; Li, D.; Chen, J.; Li, Y.; Zhoy, H.; Xie, Y. Bioorg. Med. Chem. 2009, 17, 2351. 